\title{
Intrahippocampal Transplantation of Transgenic Neural Precursor Cells Overexpressing Interleukin-I Receptor Antagonist Blocks Chronic Isolation-Induced Impairment in Memory and Neurogenesis
}

\author{
Ofra Ben Menachem-Zidon',2, Inbal Goshen', Tirzah Kreisel', Yair Ben Menahem', Etti Reinhartz², \\ Tamir Ben Hur ${ }^{2}$ and Raz Yirmiya*,I \\ 'Department of Psychology, The Hebrew University of Jerusalem, Jerusalem, Israel; ${ }^{2}$ Department of Neurology, Hadassah-Hebrew University \\ Hospital, Jerusalem, Israel
}

\begin{abstract}
The proinflammatory cytokine interleukin-I (IL-I) within the brain is critically involved in mediating the memory impairment induced by acute inflammatory challenges and psychological stress. However, the role of IL-I in memory impairment and suppressed neurogenesis induced by chronic stress exposure has not been investigated before now. We report here that mice that were isolated for 4 weeks displayed a significant elevation in hippocampal IL- I $\beta$ levels concomitantly with body weight loss, specific impairment in hippocampaldependent memory, and decreased hippocampal neurogenesis. To examine the causal role of IL-I in these effects, we developed a novel approach for long-term delivery of IL-I receptor antagonist (IL-I ra) into the brain, using transplantation of neural precursor cells (NPCs), obtained from neonatal mice with transgenic overexpression of IL-I ra (IL-I raTG) under the glial fibrillary acidic protein promoter. Four weeks following intrahippocampal transplantation of IL-IraTG NPCs labeled with PKH-26, the transplanted cells were incorporated within the dentate gyrus and expressed mainly astrocytic markers. IL-I ra levels were markedly elevated in the hippocampus, but not in other brain regions, by 10 days and for at least 4 weeks post-transplantation. Transplantation of IL-I raTG NPCs completely rescued the chronic isolation-induced body weight loss, memory impairment, and suppressed hippocampal neurogenesis, compared with isolated mice transplanted with WT cells or sham operated. The transplantation had no effect in group-housed mice. These findings elucidate the role of IL-I in the pathophysiology of chronic isolation and suggest that transplantation of IL-IraTG NPCs may provide a useful therapeutic procedure for IL-I-mediated memory disturbances in chronic inflammatory and neurological conditions. Neuropsychopharmacology (2008) 33, 225 I-2262; doi:I 0. I038/sj.npp. I 30 I606; published online 7 November 2007
\end{abstract}

Keywords: interleukin- I; chronic isolation; neurogenesis; memory; hippocampus; neural precursor cell transplantation

\section{INTRODUCTION}

Interleukin-1 (IL-1) is a pleiotropic cytokine, produced mainly by cells of the macrophage/monocyte lineage as well as by glia and neurons within the brain (Dinarello, 1996; Rothwell and Luheshi, 2000; Srinivasan et al, 2004). IL-1 is produced in two forms, IL- $1 \alpha$ and IL- $1 \beta$, whose signaling is mediated by the IL-1 receptor type I. A third member of the family, IL-1 receptor antagonist (IL-1ra), acts as a highly selective competitive antagonist (Dinarello, 1996). IL-1 receptors are localized in several areas of the rodent brain,

\footnotetext{
*Correspondence: Professor R Yirmiya, Department of Psychology, The Hebrew University of Jerusalem, Mount Scopus, Jerusalem 91905, Israel, Tel: + 9722 5883695, Fax: + 97225882947 ,

E-mail: razyirmiya@huji.ac.il

Received II September 2007; revised 19 September 2007; accepted 27 September 2007
}

with the highest densities detected in the hippocampus (Loddick et al, 1998). Brain IL-1 is induced by many immune challenges, as well as by exposure to psychological stressors, and can produce marked neural, neuroendocrine, and behavioral alterations (Besedovsky and del-Rey, 1996; Rothwell and Luheshi, 2000; Yirmiya et al, 2000).

Importantly, elevated levels of brain IL-1 were found to impair the consolidation of memories that depend on hippocampal functioning (Pugh et al, 2001; Goshen and Yirmiya, 2007). IL-1-induced memory impairments were demonstrated following intracerebroventricular (i.c.v.) and intrahippocampal administration of exogenous IL-1 (Barrientos et al, 2002), as well as the ability of IL-1ra to block the memory disturbances induced by exposure to inflammatory agents, such as lipopolysaccharide (LPS) and HIV gp120 (Pugh et al, 1998, 2000). Furthermore, exposure to acute psychological stressors, such as short-term (3-5h) isolation, increased hippocampal IL-1 levels and produced 
memory impairments that were completely blocked by an intrahippocampal injection of IL-1ra (Barrientos et al, 2003, 2004). Stressful conditions, such as social isolation, also influence several aspects of neural plasticity, including hippocampal neurogenesis (Dong et al, 2004; Westenbroek et al, 2004; Stranahan et al, 2006), which is critically important for some hippocampal-dependent learning and memory tasks (Shors et al, 2001, 2002; Saxe et al, 2006; Winocur et al, 2006). We have recently demonstrated that chronic mild stress, as well as chronic administration of IL-1 $\beta$, markedly reduces hippocampal neurogenesis (Goshen et al, 2007). However, to date the role of IL-1 in memory impairments that accompany chronic conditions has not been clearly elucidated.

Presently available methods for long-term blockade of IL-1 actions within the brain include chronic i.c.v. infusion of IL-1 ra via osmotic minipumps or intracerebral introduction of an adenoviral vector that expresses IL-1ra. However, the longest duration achieved by these methods is only 1 week (Quan et al, 2003; Depino et al, 2004). In the present study we developed a novel approach for long-term chronic blockade of brain IL-1 for at least 4 weeks, using intrahippocampal transplantation of neural precursor cells (NPCs), which have the capacity to proliferate and differentiate into all major cell types of the central nervous system (Alvarez-Buylla et al, 2001). NPCs were obtained from mice with transgenic overexpression of human IL-1 ra under the glial fibrillary acidic protein (GFAP) promoter (Lundkvist et al, 1999). We assessed the ability of transplantation of these NPCs, compared to transplantation of either NPCs from WT mice or vehicle only, to rescue the effects of chronic isolation on memory functioning and hippocampal neurogenesis.

\section{MATERIALS AND METHODS}

\section{Subjects}

Newborn transgenic mice overexpressing secreted human IL-1ra in astrocytes within the brain and spinal cord (IL-1raTG), as well as their WT C57BL/6XCBA controls (a strain derived from the nontransgenic littermates of the transgenic offspring) (Lundkvist et al, 1999; Wolf et al, 2003; Bajayo et al, 2005) were used as a source for NPCs. The subjects in the experiments were 9- to 10 -week-old WT C57BL/6 X CBA mice. Animals were housed in an air-conditioned room $\left(23 \pm 1^{\circ} \mathrm{C}\right)$, with food and water ad libitum. The experiments were conducted during the light phase of a 12-h light/dark cycle, with lights on at 8 a.m. The experiments were approved by the Hebrew University Committee on Animal Care and Use.

\section{Chronic Social Isolation Procedure and Experimental Design}

All mice were housed in groups of nine animals in a $36 \times 30 \times 15 \mathrm{~cm}$ cage, from weaning until 9-10 weeks of age. At that time, the isolated mice were housed individually in a $26.5 \times 20 \times 13.5 \mathrm{~cm}$ cage for 4 weeks while the control mice remained in their group cages.

Two isolation experiments were conducted. In the first experiment, assessing the effects of chronic isolation on memory functioning, neurogenesis, and cytokine production, 13 mice were isolated and another 13 served as grouphoused controls. Following behavioral assessments, nine mice from each group were used for assessment of hippocampal cytokine levels, and additional four mice from each group were used for analysis of hippocampal neurogenesis by doublecortin (DCX) immunohistochemistry (see below). In the second experiment, assessing the effects of NPCs transplantation on chronic isolationinduced neurobehavioral alterations, two groups of isolated or group-housed mice were each divided into further three groups (ie, a $2 \times 3$ design), including two control groups that were either sham operated or transplanted with WT NPCs and an experimental group, which was transplanted with IL-1raTG NPCs $(n=8-19$ per group). Mice were transplanted with NPCs or sham operated immediately before assigning them to the group housing or isolation conditions. Animals were killed $72 \mathrm{~h}$ after the completion of the behavioral testing. Mice (4-5) from each of these groups were used for assessment of hippocampal cytokines by enzyme-linked immunosorbent assay (ELISA), and the rest were used for immunofluorescent staining of the transplanted NPCs and analysis of hippocampal neurogenesis by DCX immunohistochemistry (see below).

\section{Measurement of Learning and Memory in the Fear-Conditioning Paradigm}

The fear-conditioning apparatus consisted of a gray colored square-conditioning cage $(30 \times 30 \times 30 \mathrm{~cm})$ with one transparent wall, to enable observation. The grid floor of the cage was wired to a shock generator and a scrambler (Coulbourn instruments, PA, USA). Mice were placed in the cage for $120 \mathrm{~s}$, and then a pure tone $(2.9 \mathrm{kHz})$ was sound for $20 \mathrm{~s}$, followed by a $2 \mathrm{~s}, 0.5 \mathrm{~mA}$ footshock. This procedure was then repeated, and $30 \mathrm{~s}$ after the delivery of the second shock mice were returned to their home cage. After $48 \mathrm{~h}$, fear conditioning was assessed by measuring the time spent in freezing (complete immobility) during exposure to the conditioned stimuli (ie, either context or the tone). The mice tested for contextual fear conditionings were placed in the original conditioning cage, and freezing was measured for $5 \mathrm{~min}$. The mice tested for the auditory-cued fear conditioning were placed in a different context - a triangular-shaped cage with no grid floor. As a control for the influence of the novel environment, freezing was measured for $2.5 \mathrm{~min}$ in this new cage, and then a $2.9 \mathrm{kHz}$ tone was sounded for $2.5 \mathrm{~min}$, during which conditioned freezing was measured. Freezing was also measured during the first $120 \mathrm{~s}$ of the conditioning trial, before the tone and shock administration, to assess possible strain differences in baseline freezing.

\section{Measurement of Learning and Memory in the Water Maze}

Animals were trained in a circular pool $(160 \mathrm{~cm}$ in diameter) filled with $23 \pm 2{ }^{\circ} \mathrm{C}$ water mixed with nontoxic gouache paint to make it opaque. In the spatial memory experiment, mice were trained to find the location of the hidden platform, submerged $1 \mathrm{~cm}$ below the surface of the pool water, using extra maze visual cues. Training consisted 
of three trials per day, with a 1-h break between trials, for 3 days. In the nonspatial memory experiment, the platform was elevated $1 \mathrm{~cm}$ above the water level and therefore was visible. Both experiments were conducted using a random protocol, in which the entrance point to the maze was varied randomly between trials, and the platform remained in a permanent position. A video camera above the pool was connected to a computerized tracking system (VP118 tracking system, HVS Image, Hampton, UK), which monitored the latency to reach the platform in each trial (see Avital et al, 2003 for additional details).

\section{Preparation of Neural Precursor Cells and Growth of Neurospheres}

Neural precursor cell spheres were prepared from newborn IL-1raTG or WT mice as previously described (Ben Hur et al, 1998, 2003). Briefly, the hemispheres were dissected, followed by removal of meninges. The tissue was minced, then digested in trypsin for $20 \mathrm{~min}$, and dissociated. Debris was removed by centrifugation through a $4 \%$ BSA layer. The cells were suspended in N2 medium, plated $10 \times 10^{6}$ cells/T75 uncoated flask, and supplemented with $10 \mathrm{ng} / \mathrm{ml}$ FGF2 and $20 \mathrm{ng} / \mathrm{ml}$ EGF, added daily. Undifferentiated neurospheres were collected for transplantation after 6 days of growth. For assessment of differentiation in vitro, floating spheres were transferred to polylysine-coated dishes, where they were allowed to adhere and differentiate for 5 days until fixated and stained for lineage-specific markers. Experiments were performed in triplicates and repeated three times, with at least 30 spheres per plate. At least 1,000 cells were counted for each experimental condition to determine cell fate.

\section{Sphere Transplantation}

Prior to transplantation, spheres were incubated with $\mathrm{PKH}-$ 26 (Sigma), a lipophilic dye that stably integrates into the cell membrane without disturbing its surface marker expression (Fischer and Mackensen, 2003). The spheres were transplanted into the hippocampus using a stereotaxic device. Coordinates of the injection sites (in $\mathrm{mm}$ ) relative to bregma were as follows: AP: $-2.6, \mathrm{~L}: \pm 1.4, \mathrm{DV}:-1.6$. Injections were conducted using a $25-\mu l$ Hamilton syringe. After each injection, the needle was left in situ for $5 \mathrm{~min}$ before being retracted to allow complete diffusion of the spheres. Each animal was injected bilaterally with a total of 6000 spheres $/ 4 \mu \mathrm{l} \mathrm{N} 2$ medium. Animals were perfused with PBS followed by $4 \%$ paraformaldehyde, 4 weeks after transplantation. Tissues were deep frozen in liquid nitrogen. Serial $8 \mu \mathrm{m}$ brain axial frozen sections were cut for histological analyses.

\section{Immunofluorescent Staining}

To determine the fate of the transplanted cells, sections were incubated with rabbit anti-GFAP $(1: 100)$ and mouse anti-neurofilament (NF) $(1: 100)$ antibodies, followed by incubation with goat anti-rabbit IgG secondary antibody and goat anti-mouse IgG secondary antibody, respectively, conjugated to Alexa-488 (Molecular Probes) for $50 \mathrm{~min}$ at room temperature (RT).

\section{Immunohistochemistry for Doublecortin}

Sections were fixated in $4 \%$ paraformaldehyde for $10 \mathrm{~min}$ at RT. After three PBS washes, sections were incubated in $3 \%$ $\mathrm{H}_{2} \mathrm{O}_{2}$ in methanol for $30 \mathrm{~min}$. After two washes with DDW and one wash with PBS, sections were incubated in $0.1 \%$ Tween in PBS for $5 \mathrm{~min}$. Sections were incubated with the primary antibody (goat anti-DCX 1:50, Santa Cruz Biotechnology) for $24 \mathrm{~h}$ at $4^{\circ} \mathrm{C}$. After $24 \mathrm{~h}$, sections were incubated with the secondary antibody (biotinylated horse anti-goat, 1:100; Vector Laboratories) for $1 \mathrm{~h}$ at RT and visualized using an avidin-biotin-peroxidase complex system (Vectastain ABC Elite Kit, Vector Laboratories). The DCX antibody labels the cell soma and its extensions, which can be visualized under the microscope as dark brown staining. The number of DCXpositive cells was evaluated by counting labeled cells on every sixth $8 \mu \mathrm{m}$ coronal section, covering the dentate gyrus in its rostrocaudal axis. The total number of cells was extrapolated for the entire volume of the dentate gyrus.

\section{Characterization of the Transplanted Cells In Vivo}

PKH-26-labeled cells were counted on every sixth $8 \mu \mathrm{m}$ coronal section, covering the dentate gyrus in its rostrocaudal extension. The total number of cells was extrapolated for the entire volume of the dentate gyrus (Kempermann et al, 1997). Differentiation of transplanted cells into astrocytes and neurons was determined by immunofluorescent staining for GFAP and for NF in brains transplanted with PKH-labeled neurospheres. The percentage of graftderived astrocytes and neurons was determined by dividing the number of the respective double-labeled cells by the number of total $\mathrm{PKH}$-positive cells. All analyses were conducted by an experimenter who was blind with respect to the experimental and control groups.

\section{RT-PCR Analysis}

Total RNA was extracted from WT and from IL-1ra transgenic NPCs using the RNeasy Kit (Qiagen). A total of $1 \mu \mathrm{g}$ RNA was subjected to reverse transcription in a $20-\mu \mathrm{l}$ reaction solution containing $500 \mathrm{ng}$ of oligo(dT)12-18, $10 \mathrm{mM}$ DTT, dNTP mix (dATP, dCTP, dGTP, and dTTP, each at $0.5 \mathrm{mM}$ ), first-strand buffer and $200 \mathrm{U}$ of M-MLV reverse transcriptase, at $42^{\circ} \mathrm{C}$ for $1 \mathrm{~h}$. cDNA $(3 \mu \mathrm{l})$ was used for the PCR analysis. PCR was performed for 35 cycles $\left(94^{\circ} \mathrm{C}\right.$ for $1 \mathrm{~min}, 55^{\circ} \mathrm{C}$ for $1 \mathrm{~min}$, and $72^{\circ} \mathrm{C}$ for $\left.1 \mathrm{~min}\right)$. The following primers were used: $\beta$-actin: TTGTAACCAACTGGGACGA TATGC (+), GATCTTGATCTTCATGATGCTAGG (-); FGF2: AAGCGGCTCTACTGCAAG $(+)$, AGCCAGACATTGGAA GAAACA (-); CNTF: GGAAGATTCGTTCAGACCTGAC $(+)$, CCCATCAGCCTCATTTTCAGGG (-); TGF- $\alpha$ : GTGGTGT CTCACTTCAACAAGT $(+)$, GACCACTGTCTCAGAGTGGC (-); BDNF: CCAGAGCAGCTGCCTTGATG $(+)$, TCTTCCCCT TTTAATGGTCAGTG (-); NGF: CTGGATGGCATGCTGG ACC $(+)$, GATTGTACCATGGGCCTGGAA $(-)$.

\section{Sample Preparation and Enzyme-Linked Immunosorbent Assay}

After the completion of the behavioral tests, the animals were killed by decapitation, brains were removed, and the 
frontal cortex, amygdala, hippocampus and hypothalamus were rapidly dissected and frozen in $-70^{\circ} \mathrm{C}$ for later analysis. At the beginning of the immunological analyses, samples were thawed, placed in cold RPMI 1640 containing $100 \mathrm{~K} I U$ aprotinin, homogenized, and centrifuged for $15 \mathrm{~min}$ at $4^{\circ} \mathrm{C}$. Hippocampal IL- $1 \beta$, IL-6, and brain-derived neurotrophic factor (BDNF) levels were determined using murine ELISA kits (R\&D Systems, Minneapolis, MN). The reported sensitivity of these assays is $3.0,1.6$, and $20 \mathrm{pg} / \mathrm{ml}$, respectively. The intra-assay coefficient is $4.4,4.8$, and $4.1 \%$ and the inter-assay coefficient is $6.1,7.5$, and $9.3 \%$, respectively. Human IL-1ra (hIL-1ra) levels derived from the transgenic NPCs were determined in the hippocampus, frontal cortex, amygdala, and hypothalamus using an hIL1 ra ELISA kit (R\&D Systems). The reported sensitivity of this assay is $14 \mathrm{pg} / \mathrm{ml}$, the intraassay coefficient is $5.5 \%$, and the interassay coefficient is $5.4 \%$. No crossreactivity was found between the hIL-1ra and the endogenous murine IL$1 \mathrm{ra}$, ie the levels of hIL-1ra in mice that were not transplanted with hIL-1raTG NPCs were close to 0 . Total protein was determined by the Bradford method, and ELISA results were normalized accordingly.

\section{Statistical Analysis}

The results were analyzed by $t$-tests or two-way ANOVAs, followed by the Tukey's HSD post hoc analysis, when appropriate.

\section{RESULTS}

\section{Chronic Isolation Induces Memory Disturbances}

Social isolation for 4 weeks caused a significant $38 \%$ elevation in hippocampal IL-1 $\beta$ levels, as compared to group-housed control mice $\left(t_{12}=4.57, p<0.05\right)$ (Figure 1a). In contrast, there was no difference in IL-6 levels between the isolated and group-housed mice $(p>0.1)$ (Figure 1b). The isolated mice displayed significantly lower body weight $\left(t_{9}=-4.8, \quad p<0.001\right)$ than group-housed controls (Figure 1c), attesting to the stressful nature of this manipulation. However, there were no differences between the isolated and group-housed controls in plasma corticosterone levels $(p>0.1)$ (Figure 1d).

Isolated mice displayed significant memory impairment in the contextual fear-conditioning paradigm $\left(t_{16}=2.89\right.$, $p<0.05$ ) (Figure 1e), whereas their auditory-cued memory functioning was comparable to group-housed controls $(p>0.1)$ (Figure 1f). Neurogenesis was evaluated by counting the number of DCX-labeled cells in the dentate gyrus of group-housed (Figure $1 \mathrm{~h}$ ) and isolated mice (Figure 1j). Isolated mice exhibited a significant $45 \%$ reduction in neurogenesis, as compared to group-housed mice $\left(t_{8}=10.73, p<0.001\right)$ (Figure $\left.1 \mathrm{~g}\right)$. Because previous studies demonstrated that acute social isolation, as well as IL-1 administration, reduces the production of BDNF (Barrientos et al, 2003; Scaccianoce et al, 2006), which is involved in both memory processes and neurogenesis (Lee et al, 2002; Heldt et al, 2007), we also measured BDNF levels within the hippocampus. However, hippocampal BDNF levels were similar in isolated and group-housed mice $(1548 \pm 134$ and $1349 \pm 102 \mathrm{pg} / \mathrm{ml}$, respectively) $(p>0.1)$.

\section{Characterization of IL-1raTG and WT NPCs In Vitro}

Neural precursor cells from newborn WT and IL-1raTG mice were expanded in spheres. Efficiency of IL-1raTG neurosphere generation from dissociated brains and their growth was similar to that of WT mice (data not shown). The potential of NPCs to differentiate in vitro along the three major neural lineages was examined by immunofluorescent staining, after 5 days of differentiation. Similar to our previous reports, IL-1raTG neurospheres comprised mostly astrocytes ( $80 \pm 3 \%$ of all differentiated cells). Figure 2a presents one sphere that is mainly positive to GFAP. A higher magnification of GFAP-positive cells from the same sphere can be visualized in Figure $2 \mathrm{~b}$. In addition, $17 \pm 4 \%$ of the cells differentiated into oligodendrocytes and only $3 \pm 1 \%$ differentiated into neurons. Similar finding were obtained in WT neurospheres, which comprised $80 \pm 4 \%$ astrocytes, $16 \pm 3 \%$ oligodendrocytes, and $4 \pm 1 \%$ neurons. Thus, IL-1raTG NPCs showed similar pattern of growth and lineage choice as WT NPCs.

To evaluate the ability of the cultured transgenic cells to secrete hIL-1ra, the culture medium was collected and hIL1ra levels were measured by ELISA. The medium from the differentiated IL-1raTG NPCs contained high levels of hILra, whereas no hIL-1ra could be detected in medium from undifferentiated IL-1raTG NPCs or differentiated WT NPCs $\left(\mathrm{F}_{2,9}=794.4, p<0.001\right)$ (Figure $2 \mathrm{c}$ ).

Because the overexpression of IL-1ra by NPCs could potentially act in an autocrine manner to alter the expression of growth and neurotrophic factors, which might be relevant to the neurobehavioral effects of these cells, we measured the expression of several such factors in IL-1raTG and WT NPCs. Expression patterns were determined under two conditions: floating undifferentiated spheres and cells after differentiation. mRNA levels were measured using a semi-quantitative RT-PCR analysis. We found that WT and IL-1raTG NPCs exhibited similar patterns of expression of FGF2, CNTF, TGF- $\alpha$, NGF, and BDNF, both before and after differentiation (data not shown). Moreover, ELISA determination of BDNF secretion was similar in cultures of differentiated WT and IL-1raTG NPCs $(p>0.1)$ (Figure 2d).

\section{Transplantation and Characterization of IL-1raTG NPCs In Vivo}

To obtain long-term production of IL-1ra in the hippocampus we transplanted IL-1raTG cells directly into the hippocampus. Since transplanted NPCs (and especially in the form of spheres) integrate and survive in the adult brain better than mature cells (Svendsen et al, 1998; Pluchino et al, 2007), we grafted undifferentiated NPC spheres. The survival, distribution, and differentiation of transplanted cells labeled with $\mathrm{PKH}-26$ were examined after completion of the behavioral tests. NPCs were localized within the hippocampus. The tract of the cannula used for graft transplantation, ending at the hippocampus, can be seen (Figure 2e-g). Transplanted cells in the dentate gyrus of the hippocampus are marked with arrowheads. Along the transplantation tract, cells were also apparent in the cortex, however, in adjacent anterior and posterior sections the transplanted cells were located only in the hippocampus. 

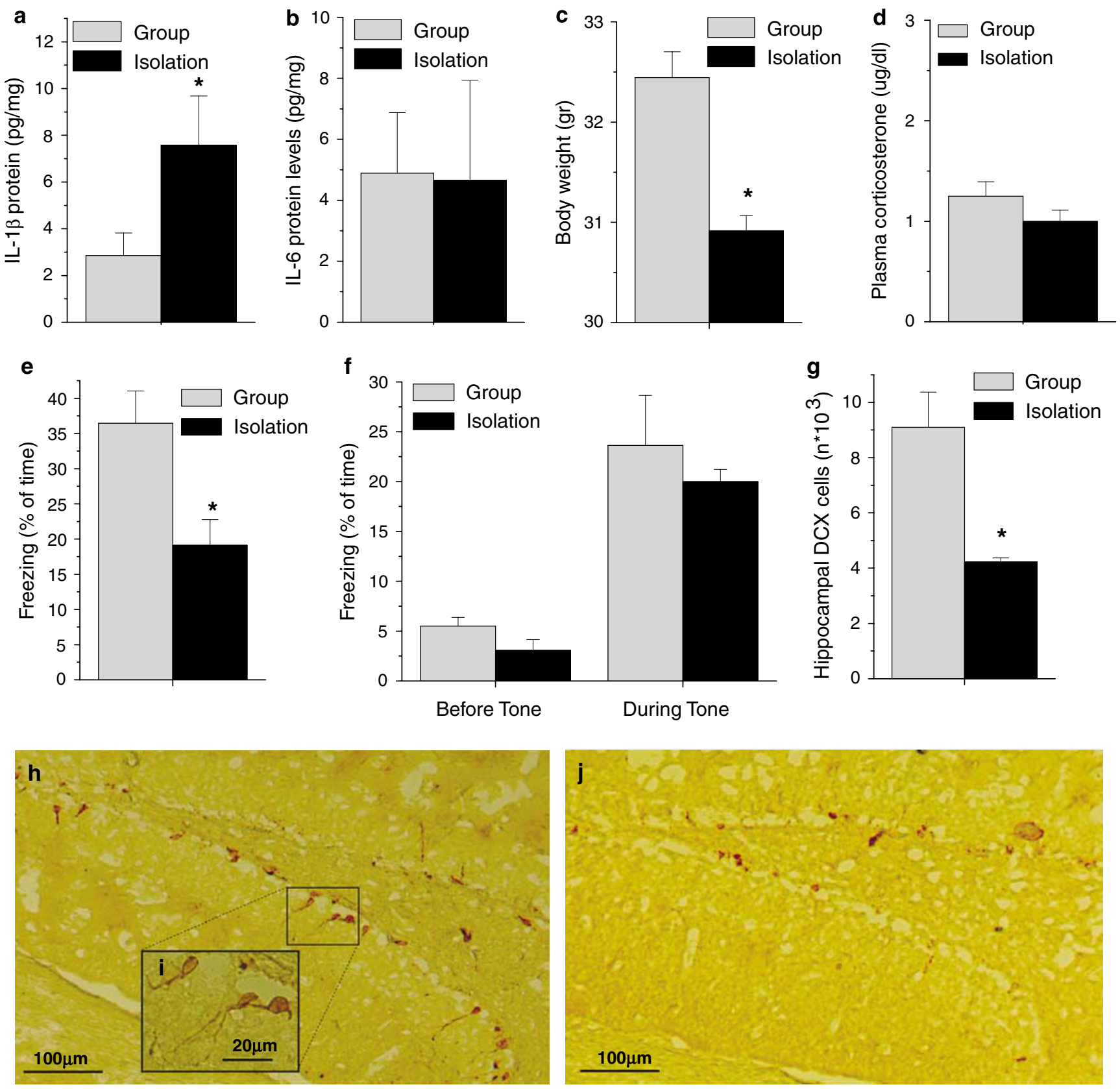

Figure I Chronic isolation induces memory disturbances and decline in hippocampal neurogenesis. Compared to group-housed control mice ( $n=9)$, mice that were isolated for 4 weeks $(n=9)$ displayed significantly increased hippocampal IL-I $\beta$ protein levels $(a)$, no change in the levels of hippocampal IL-6 levels (b), a significant decrease in body weight (c), and no change in plasma corticosterone levels (d). In addition, isolated mice displayed impaired contextual memory in the fear-conditioning paradigm (which depends on normal hippocampal functioning) (e), but no impairment in auditory-cued memory, which is hippocampus independent (f). Chronic isolation caused a significant decline in the number of doublecortin (DCX)-positive cells in the dentate gyrus (DG) of the hippocampus (g). Normal levels of neurogenesis in the DG of the hippocampus were observed in group-housed mice $(n=4)$, reflected by relatively high number of new neurons, which express the early neuronal marker DCX (stained in dark brown) (h). A higher magnification of the DCX staining within the black square shows the typical morphology of DCX-positive neurons with their extensions (i). A smaller number of DCX-positive cells were observed in the DG of isolated mice $(n=4)$ (j). Data are presented as mean \pm SEM. $* p<0.05$, compared to group-housed mice.

Most of the cells were localized in the dentate gyrus and were integrated into the host parenchyma (Figure $2 \mathrm{~h}$ ). Confocal microscopy in the sections containing PKH-26labeled cells showed that the labeling was retained in the nucleus (Figure $2 \mathrm{i}$ and $\mathrm{j}$ ). To evaluate the number of surviving transplanted cells, as well as the effects of isolation on their survival, we counted the number of
PKH-labeled cells within the hippocampus. The average $( \pm$ SEM) number of PKH-labeled cells in isolated WT NPCs-transplanted mice, and IL-1raTG NPCs-transplanted mice was $5500 \pm 345$ and $5200 \pm 350$, respectively. These numbers were similar to the respective group-housed groups ( $5443 \pm 300$ and $5100 \pm 250$ ), indicating no effect of the isolation procedure on the NPCs survival. 

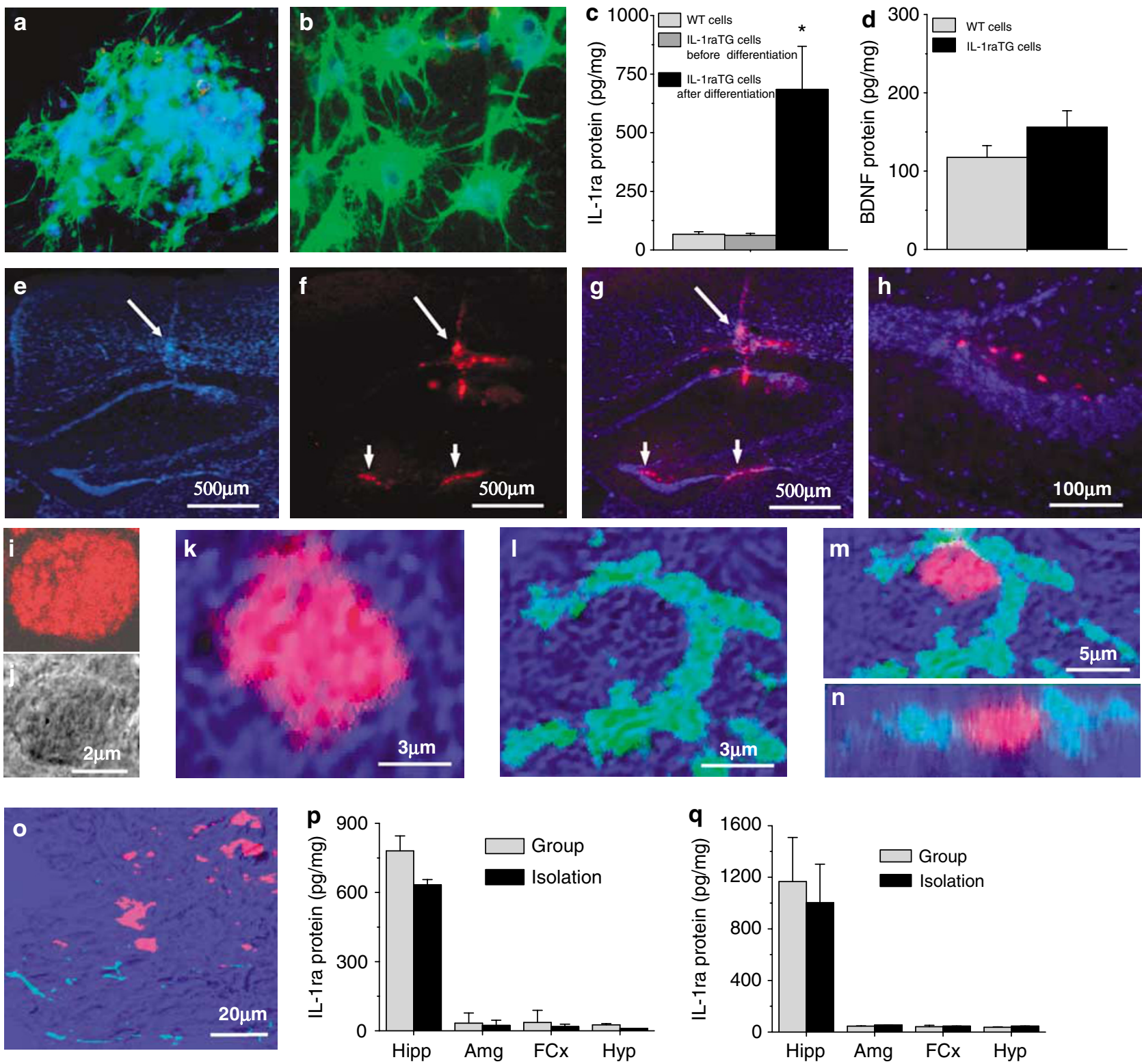

Figure 2 Characterization of IL-IraTG and WT NPCs. NPCs were first characterized by immunofluorescent staining, performed after 5 days of differentiation in vitro. A representative picture of one sphere demonstrates that most of the cells (blue = Dapi) differentiated into astrocytes, expressing GFAP (green = GFAP) (a). A magnification of GFAP-positive cells from the same sphere can be seen in (b). The production of hlL-I ra by NPCs in vitro was examined just before and following differentiation for 5 days. High levels of hIL-I ra were found only in the medium above differentiated IL-I raTG cultures (c). There was no difference in the levels of brain-derived neurotrophic factor (BDNF) secreted from IL-I raTG and WT differentiated NPCs (d) The transplantation tract can be seen in a coronal section of the hippocampus, counterstained with the nuclear tracer Dapi (e, arrow) ) 4-5 weeks following transplantation of NPCs into the host's hippocampus. Transplanted PKH-26-labeled cells (in red) can be visualized along the needle tract entering the hippocampus (f, arrow) and within the DG (f, arrowheads). A few PKH-26-labeled cells were observed in the cortex, only at the site of needle penetration. A merged image of e and $\mathrm{f}$ shows the PKH-26-labeled cells along the needle tract and in the hippocampus, counterstained with Dapi ( $\mathrm{g}$ ). At a higher magnification, the PKH-26-labeled transplanted cells can be clearly seen in the subgranular zone of the host DG (h). Confocal microscopy in the same section showed that the PKH-26 labeling (i) was limited to the nucleus, as indicated by Nomarsky optics (j). Confocal microscopy showed that transplanted PKH-26-labeled nuclei ( $\mathrm{k}$ ) were surrounded by GFAP + cytoplasm (I). The colocalization of PKH-26 and GFAP within the same cell is shown in the merged image $(\mathrm{m})$ and $z$ axis $(\mathrm{n})$. However, no colocalization of PKH-26-labeled cells could be found with the neuronal marker NF 200 (o). hIL-I ra levels were examined in the hippocampus (Hipp), amygdala (Amg), frontal cortex (FCX) and hypothalamus (Hyp) of group-housed and isolated mice that were transplanted with IL-I raTG NPCs. High levels of hIL-I ra were found only in the hippocampus, both at I 0 days post-transplantation (p), and 4-5 weeks posttransplantation (q). * $p<0.05$ vs WT and IL-I raTG nondifferentiated NPCs.

To evaluate the pattern of differentiation of the WT and IL-1raTG-transplanted cells, slides with PKH-labeled cells were immunostained with glial and neuronal markers. In accordance with previous work (Hovakimyan et al, 2006), only a fraction of transplanted cells differentiated and acquired lineage-specific cell markers. In both isolated and group-housed mice, about $18 \%$ of the WT and IL-1raTG-transplanted PKH cells differentiated into 
astrocytes, expressing GFAP (Figure $2 \mathrm{k}$ and 1). Confocal microscopy images confirmed the coexpression of GFAP and $\mathrm{PKH}-26$ (Figure $2 \mathrm{~m}$ and $\mathrm{n}$ ). The possibility that some of the transplanted cells differentiated into neurons was examined by immunofluorescence staining for the neuronal marker anti-heavy chain NF 200. None of the transplanted cells were positive for this marker (Figure 20).

\section{Production of hIL-1ra by IL-1raTG NPCs In Vivo}

To examine the ability of the transplanted cells to produce hIL-1ra in vivo, and to evaluate the time course of this production, levels of hIL-1ra protein were measured by ELISA at 10 days and at 4-5 weeks post-transplantation. These time point were chosen because they represent an early phase in which the cells are known to incorporate and differentiate within the host brain, and a late phase in which the cells have survived in the host brain throughout the experimental period. To examine the possible diffusion of hIL-1ra from the hippocampus we measured the levels of hIL-1ra in this structure, as well as in several other brain regions that are involved in the behavioral and cognitive effects of IL-1, including the amygdale, frontal cortex, and hypothalamus. High levels of hIL-1ra were found in the hippocampus, but not in any other brain region, both at 10 days post-transplantation $\left(\mathrm{F}_{2,23}=123.5, \quad p<0.001\right)$ (Figure 2p) and at 4-5 weeks post-transplantation $\left(\mathrm{F}_{2,19}=112, p<0.001\right)$ (Figure $\left.2 \mathrm{q}\right)$, suggesting that the transgenic IL-1ra production remains fairly localized within the hippocampus. No difference was found between isolated and group-housed mice in hIL-1ra production, which together with our histological findings demonstrate that chronic isolation had no effect on NPCs survival, differentiation, and functionality. The levels of IL- 6 in either isolated or group-housed mice were not altered by NPCs transplantation (data not shown).

\section{Effects of hIL-1raTG NPCs Transplantation on Learning and Memory}

In the fear-conditioning paradigm, isolated sham-operated and WT NPCs-transplanted mice displayed reduced contextual fear conditioning compared to their respective group-housed controls, whereas transplantation of hIL1raTG NPCs completely rescued the chronic isolationinduced memory impairments (Figure $3 \mathrm{a})\left(\mathrm{F}_{2,53}=4.493\right.$, $p<0.05)$. The transplantation had no effect on contextual fear conditioning in group-housed mice. In the auditorycued fear-conditioning paradigm, neither the chronic isolation nor the transplantation had an effect on freezing, either before the tone presentation (data not shown) or during the tone (Figure $3 \mathrm{~b}$ ).

In the water maze paradigm, isolated mice transplanted with WT NPCs displayed severely impaired spatial memory, with almost no reduction in the latencies to reach the hidden platform over the 3 training days, whereas isolated mice transplanted with IL-1raTG NPCs acquired the spatial memory task similarly to group-housed mice $\left(\mathrm{F}_{3,42}=8.185, p<0.001\right.$ ) (Figure $\left.3 \mathrm{c}\right)$. No difference was found between the three groups in the nonspatial paradigm (data not shown).

\section{Effects of hIL-1raTG NPCs Transplantation on Hippocampal Neurogenesis and BDNF Production}

Immunohistochemistry for DCX in the dentate gyrus revealed that isolated sham-operated mice or mice transplanted with WT cells displayed suppressed hippocampal neurogenesis, compared with their respective group-housed controls, and this effect was completely abolished in mice transplanted with hIL-1raTG NPCs $\left(\mathrm{F}_{2,53}=4.493, p<0.05\right)$ (Figure $3 \mathrm{~d}$ ). The transplantation had no effect on neurogenesis in nonisolated mice. There was no difference in hippocampal BDNF levels between isolated and grouphoused mice transplanted with WT or IL-1raTG NPCs or sham operated $(p>0.1)$ (Figure $3 e)$.

\section{Effects of hIL-1raTG NPCs Transplantation on Body Weight Loss}

Body weight was measured at baseline, as well as following the behavioral testing. All group-housed mice gained weight during this period, whereas isolated mice that were sham operated or transplanted with WT NPCs displayed significant weight loss compared with their respective controls, demonstrating the stressful nature of this manipulation. In contrast, isolated mice that were transplanted with IL-1 raTG NPCs did not differ from their respective group-housed controls and displayed significantly more weight gain than the two other isolated groups $\left(F_{3,14}=10.6, p<0.05\right)$ (Figure 3f).

\section{DISCUSSION}

The results of the present study demonstrate that subjecting mice to chronic isolation markedly elevated the levels of IL$1 \beta$, but not IL- 6 , within the hippocampus, concomitantly with impairment in hippocampal-dependent learning and memory, suppression of neurogenesis, and body weight loss. Chronic blockade of IL-1 signaling by intrahippocampal transplantation of IL-1raTG NPCs, which chronically elevated the levels of IL-1ra throughout most of the isolation exposure period, completely abolished these detrimental effects. These results demonstrate, for the first time, a causal relationship between the effects of chronic isolation on hippocampal IL-1 levels and memory impairments, and suggest that this relationship involves IL-1-mediated chronic isolation-induced suppression of hippocampal neurogenesis.

In the present study we used 4-week social isolation as a model for studying the effects of chronic stress on memory functioning and neurogenesis. Our findings that chronic isolation significantly impaired contextual fear conditioning and spatial memory, but not auditory-cued fear conditioning or nonspatial performance in the water maze are consistent with previous reports that either acute (Barrientos et al, 2003) or chronic (Kogan et al, 2000; Bianchi et al, 2006) isolation markedly impaired hippocampal-dependent memory, but not memory that does not depend on hippocampal functioning. Previous studies on the effects of chronic stress on hippocampal neurogenesis demonstrated decreased cell proliferation in the dentate gyrus following chronic isolation (Dong et al, 2004), as well as several other chronic stressors (Simon et al, 2005; Lee et al, 

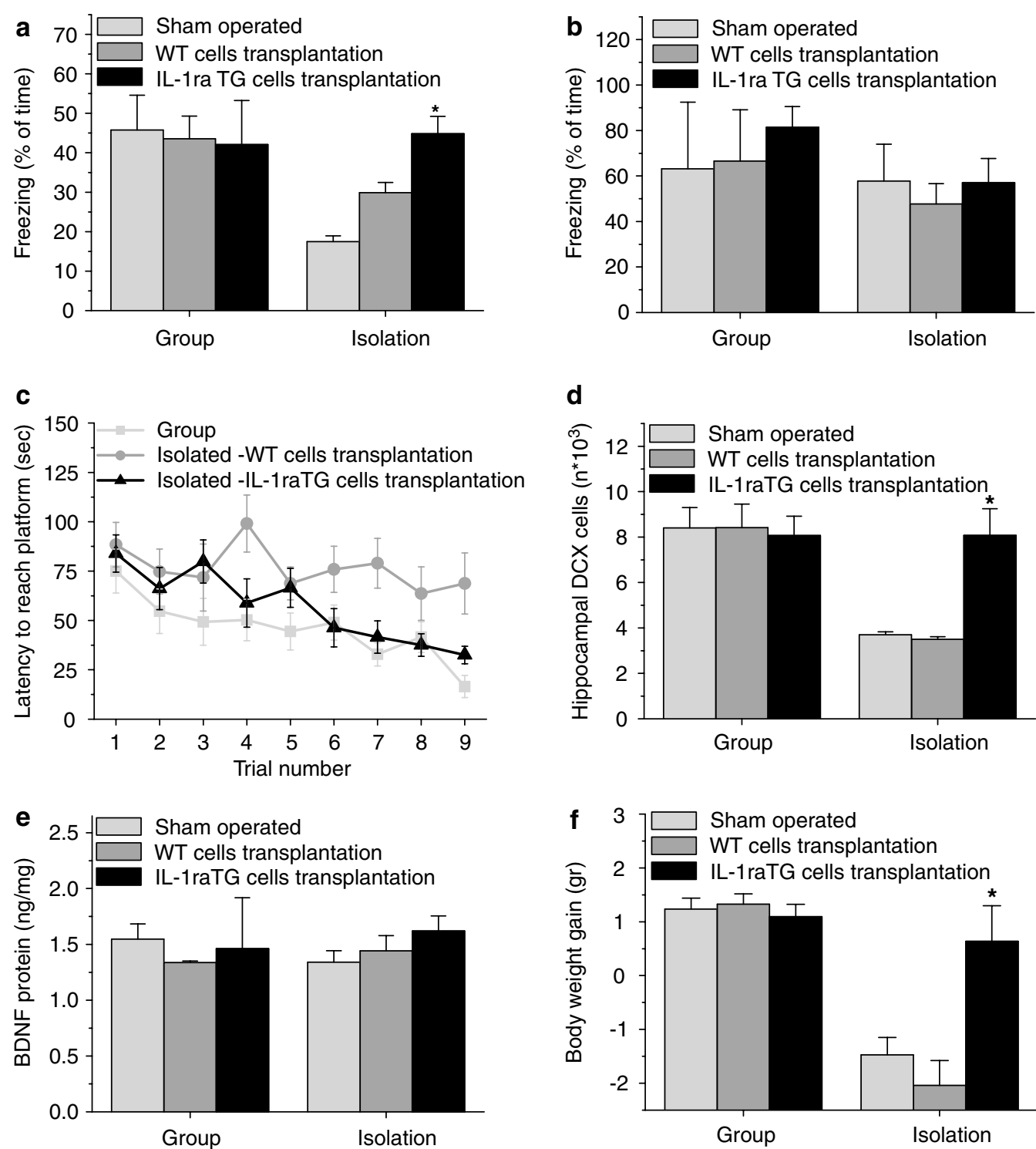

Figure 3 Transplantation of mice with transgenic overexpression of IL-I ra (IL-I raTG) NPCs rescues chronic isolation-induced impairments in memory and neurogenesis. (a) In the fear-conditioning paradigm, isolated sham-operated and WT NPCs-transplanted mice displayed reduced conditioned fear (freezing) compared to their respective group-housed controls, whereas in isolated mice transplanted with hIL-I raTG NPCs the chronic isolation-induced memory impairment was completely rescued. The transplantation had no effect in nonisolated, group-housed mice. (b) In contrast, in the auditory-cued fearconditioning paradigm, neither the isolation nor the transplantation had an effect on freezing. (c) In the water maze paradigm, isolated mice transplanted with WT NPCs displayed severely impaired spatial memory, with almost no reduction in the latencies to reach the hidden platform over the 3 training days (with three trials per day), whereas isolated mice transplanted with IL-IraTG NPCs acquired the spatial memory task similarly to group-housed mice. (d) Immunohistochemistry for doublecortin (DCX) revealed that isolated sham-operated or WT NPCs-transplanted mice displayed significantly reduced hippocampal neurogenesis, whereas transplantation of hIL-I raTG NPCs completely rescued the suppressive effect of isolation. The NPCs transplantation had no effect in nonisolated mice. (e) Hippocampal brain-derived neurotrophic factor (BDNF) levels were not affected by either isolation or NPCs transplantation. (f) Body weight was measured at baseline, as well as following the behavioral testing (4-5 weeks post-transplantation). All group-housed mice gained weight during this period, whereas isolated sham-operated or WT NPCs-transplanted mice displayed significant weight loss. In contrast, isolated mice that were transplanted with IL-I raTG NPCs did not differ from their respective group-housed controls. Data are presented as mean \pm SEM. * $p<0.05$ compared to the other groups of isolated mice.

2006; Goshen et al, 2007). Chronic social isolation also exacerbated the effect of another chronic stressor (daily foot-shock for 3 weeks) on the production of new hippocampal neurons (Westenbroek et al, 2004), and precluded the positive influence of short-term running on neurogenesis (Stranahan et al, 2006). Our findings are consistent with these reports, demonstrating that chronic isolation decreased the production of new neurons, which has been implicated in some hippocampal-dependent learning and memory tasks (Shors et al, 2001; Shors, 2004; Saxe et al, 2006). Thus, it can be suggested that chronic isolation-induced reduction in neurogenesis may be involved in the memory impairment in contextual fear conditioning and spatial memory that accompanies this condition.

Despite its marked effects on memory and neurogenesis, chronic isolation had no effect on the production of 
hippocampal BDNF. This finding does not corroborate previous research, which demonstrated that BDNF is an important modulator of hippocampal-dependent memory formation (Heldt et al, 2007) and neurogenesis (Lee et al, 2002), and that acute isolation stress produces an IL-1mediated decrease in hippocampal BDNF expression and memory functioning (Barrientos et al, 2003). This discrepancy could stem from a procedural difference, because in contrast to all of the above-mentioned studies, in which BDNF levels were measured shortly after behavioral testing, in the present experiments the animals were killed $72 \mathrm{~h}$ after the end of the behavioral testing. It is possible that by that time the levels of BDNF had already returned to baseline. Another explanation for the discrepant results could be that in contrast with the lack of corticosterone elevation found in the present study, acute isolation induces marked corticosterone secretion (Stranahan et al, 2006), which is known to suppress BDNF production (Jacobsen and Mork, 2006). However, a previous study on the effects of chronic (8-week) isolation demonstrated a significant reduction in hippocampal BDNF protein levels in the absence of corticosterone elevation (Scaccianoce et al, 2006). The difference between these results and ours could be due to the use of a different mouse strains.

Ample research indicates that exposure to acute stressors, such as footshock or $5 \mathrm{~h}$ isolation, induces the production of IL-1 in the hippocampus (Pugh et al, 1999; O'Connor et al, 2003). Most studies on the effects of chronic stressors on the IL-1 system focused on the mRNA level and their results are less clear (Plata-Salaman et al, 2000; Bartolomucci et al, 2003). However, consistently with the present finding, we have recently discovered that exposure to chronic mild stress induces a significant increase in hippocampal IL-1 $\beta$ protein levels (Goshen et al, 2007). Stress-induced elevation in hippocampal IL- $1 \beta$ levels could potentially mediate the memory impairment exhibited by the socially isolated mice, because previous research demonstrated that IL-1 administration, either peripherally (Pugh et al, 1998), intracerebroventricularly (Pugh et al, 2001), or directly into the hippocampus (Barrientos et al, 2002), impairs contextual, but not auditory-cued fear conditioning. Consistently, administration of IL-1ra has been previously reported to block the behavioral and neuroendocrine effects of acute stress (Pugh et al, 1998, 2000). Isolation-induced suppressed neurogenesis was probably also mediated by IL-1, consistent with our recent finding that chronic mild stress suppressed hippocampal neurogenesis in WT mice, but not in mice with deletion of the IL-1 receptor, and that chronic administration of IL- $1 \beta$ (via osmotic minipumps) also produced a marked reduction in this process (Goshen et al, 2007). These finding are also consistent with reports that treatment with IL-1 inducers, including LPS and radiation, results in marked suppression of hippocampal neurogenesis (Ekdahl et al, 2003; Monje et al, 2003).

To directly assess the causal role of chronic isolationinduced IL-1 production in memory impairment and suppressed neurogenesis we devised a novel approach for chronic blockade of IL-1 signaling by intra-hippocampal transplantation of NPCs from IL-1raTG mice. The findings that these cells could be visualized within the hippocampus and that the levels of IL-1ra in the hippocampus were elevated even 4-5 weeks post-transplantation validate the effectiveness of this method. In agreement with previous reports (Ben Hur et al, 1998; Cao et al, 2001), most NPCs differentiated into astrocytes and not neurons, and seemed to be integrated in the parenchyma of the hippocampal dentate gyrus. Previously, administration of IL-1ra into the brain was achieved by infusion of IL-1ra through a chronically implanted cannula (Schneider et al, 1998; Quan et al, 2003) or through a recombinant adenovirus encoding IL-1ra (Depino et al, 2004). Using each of these methods as a therapeutic procedure for long-term IL-1ra administration has important drawbacks. The i.c.v. route is problematic because implantation of the i.c.v. cannula induces a chronic inflammatory condition associated with high levels of endogenous IL-1 expression and greater IL-1 responsiveness to subsequent inflammatory challenges (Holguin et al, 2007). Furthermore, IL-1ra has a short half-life, so repeated administration is required (this problem can be circumvented by using continuous infusion via osmotic minipumps). The use of vectors is also problematic, as it requires several steps until high levels of expression are achieved, and there are cytotoxic side effects (Hagan et al, 1996; Depino et al, 2004). These considerations may explain the paucity of research on chronic blockade of brain IL-1 and the fact that to date the maximal period of such blockade has been only 7 days (Quan et al, 2003; Depino et al, 2004). Our method of transplanting genetically engineered NPCs seems to circumvent some of these problems, allowing for stable expression over time and enabling long-term local secretion of IL-1ra within a specific microenvironment.

The results demonstrate that in the fear-conditioning paradigm as well as in the Morris water maze, isolated mice that were transplanted with IL-1raTG NPCs displayed better memory compared to animals that received NPCs from WT mice. The difference between the two groups can be attributed only to the secretion of IL-1ra within the hippocampus of the transplanted animals, demonstrating that elevated levels of IL-1 play a critical role in the memory impairment induced by exposure to chronic stressful conditions. The small beneficial effect that was apparent in the mice transplanted with WT NPCs could have resulted from nonspecific effects of growth factors secreted by these cells. Indeed, it has been reported that transplantation of astrocytes could improve cognitive deficits (Bradbury et al, 1995; Iguchi et al, 2003).

An acute inflammatory response is expected following the insertion of the cannula for cell transplantation (as well as sham operation). However, based on our own experience (comparing the functioning of sham-operated to naive mice) and on a recent systematic investigation of hippocampal cannulation-induced local inflammation (Holguin et $\mathrm{al}, 2007$ ), the effect of an acute insertion of a cannula into the hippocampus on inflammatory responses (including IL$1 \beta$ production and its responsiveness to subsequent immune challenge) lasts less than 1 week, and therefore is not expected to produce a significant effect on memory functioning in the present study.

We have recently discovered that IL- $1 \beta$ expression is specifically induced following fear-conditioning training (Goshen et al, in press). We also found that acute injections of IL-1ra ( $100 \mu \mathrm{g}$, i.c.v.) immediately following learning acquisition, as well as chronic exposure to IL-1ra (either in 
mice with transgenic overexpression of brain IL-1ra or in WT mice with chronic infusion of IL-1ra by osmotic mini pumps) significantly impaired memory functioning in the passive avoidance, water maze, and fear-conditioning paradigms, attesting to the important role of physiological levels of brain IL-1 for memory consolidation (Yirmiya et al, 2002; Goshen et al, 2007). These findings seem to contrast with the results of the present study, in which transplantation of IL-1raTG cells had no effect on memory functioning in WT group-housed mice. However, it should be appreciated that the physiological effects of IL-1 depend on the balance between IL-1 and IL-1ra (Goshen and Yirmiya, 2007; Goshen et al, in press). Thus, it is possible that in previous studies the acute or chronic exposure to globally high doses of IL-1ra throughout the hippocampus produced a more complete blockade of all IL-1 receptors, which did not allow the physiological effect of IL-1 on memory consolidation, whereas in the present study the local elevation of IL-1ra level in the transplanted mice blocked the detrimental effects of stress-induced IL-1, but still allowed some IL-1 at hippocampal regions that are not adjacent to the transplanted NPCs to interact with its receptors and produce its beneficial effects on memory consolidation. In addition, it is possible that the consolidation-enabling effects of learning-induced IL-1 production (Goshen et al, in press) are exerted not only within the hippocampus and therefore they were blocked by the global pharmacological or genetic manipulations in brain IL-1ra, but not by local infusion of IL-1ra into the hippocampus in the transplanted mice.

In addition to its beneficial effects on cognitive functioning and neurogenesis, transplantation of IL-1 raTG cells also completely rescued the chronic isolation-induced body weight loss. Chronic stressors in general and isolation in particular, are known to induce body weight loss (Guo et al, 2004), which is related to alterations in food intake, and other metabolic processes. Brain IL-1 has been shown to produce marked effects on body weight and food intake (Avitsur et al, 1997) and to mediate the effects of other challenges on these parameters (Yirmiya et al, 1999). However, to our knowledge no previous studies attempted to assess the specific role of the hippocampus in IL-1mediated anorexia and body weight loss. Nevertheless, it should be noted that the hippocampus has been proposed to participate in body weight regulation (Davidson et al, 2005), possibly via receptors to several feeding and body weightregulating hormones, including leptin, insulin, and glucocorticoids (Wada et al, 2005; Harvey, 2003), which in turn interact with the hippocampal IL-1 system (Hosoi et al, 2002; Dinkel et al, 2003). Moreover, the anorexic response to chronic i.c.v. infusion of IL-1 $\beta$ was associated with elevations in hippocampal IL-1 $\beta$ levels (Gayle et al, 1997).

Excessive production and secretion of IL-1 within the brain have been implicated in several medical conditions, including multiple sclerosis (MS), ischemic injury, and neurodegenerative diseases, particularly Alzheimer's disease (Griffin and Mrak, 2002; Allan et al, 2005; Moynagh, 2005). Indications that some of the symptoms associated with these conditions are mediated by IL-1 include elevated levels of IL-1 in several brain areas (Lynch, 2002; Pollak et al, 2003a; Griffin and Mrak, 2002), the fact that some of the pathological findings associated with these conditions (e.g. memory impairments) can be mimicked experimentally in animals by IL-1 administration (Pugh et al, 2001; Goshen and Yirmiya, 2007), and the ability of treatment with agents that inhibit the action of IL-1 to reduce some of the pathological symptoms induced in clinical settings and experimental animal models of these medical conditions (Pollak et al, 2003b; Emsley et al, 2005). The results of the present study demonstrate that chronic exposure to isolation can also be considered an IL-1-mediated condition. These findings may be relevant to previous reports that chronic stress can exacerbate the symptoms of other IL-1-mediated medical conditions, including Alzheimer's disease and MS (Wilson et al, 2003; Brown et al, 2006; Jeong et al, 2006). Long-term suppression of IL-1 signaling should be highly beneficial for any medical condition associated with excessive brain IL-1 production. Thus, suitability of our method of transplanting NPCs overexpressing IL-1ra for chronic medical conditions other than chronic isolation, particularly autoimmune disorders, such as MS, and neurodegenerative diseases, such as Alzheimer's disease, should be investigated further.

\section{ACKNOWLEDGEMENTS}

We thank Prof. K Iverfeldt for the IL-1raTG mice. This study was supported by grants from the Israel Ministry of Health (no. 2985) and the Israel Science Foundation (Grant no. $295 / 07$ to RY). RY is a member of the Eric Roland Center for Neurodegenerative Diseases at the Hebrew University of Jerusalem.

\section{DISCLOSURE/CONFLICT OF INTEREST}

The author(s) declare that except for income received from my primary employer no financial support or compensation has been received from any individual or corporate entity over the past 3 years for research or professional service and there are no personal financial holdings that could be perceived as constituting a potential conflict of interest.

\section{REFERENCES}

Allan SM, Tyrrell PJ, Rothwell NJ (2005). Interleukin-1 and neuronal injury. Nat Rev Immunol 5: 629-640.

Alvarez-Buylla A, Garcia-Verdugo JM, Tramontin AD (2001). A unified hypothesis on the lineage of neural stem cells. Nat Rev Neurosci 2: 287-293.

Avital A, Goshen I, Kamsler A, Segal M, Iverfeldt K, Richter-Levin $\mathrm{G}$ et al (2003). Impaired interleukin-1 signaling is associated with deficits in hippocampal memory processes and neural plasticity. Hippocampus 13: 826-834.

Avitsur R, Pollak Y, Yirmiya R (1997). Administration of interleukin-1 into the hypothalamic paraventricular nucleus induces febrile and behavioral effects. Neuroimmunomodulation 4: 258-265.

Bajayo A, Goshen I, Feldman S, Csernus V, Iverfeldt K, Shohami E et al (2005). Central IL-1 receptor signaling regulates bone growth and mass. Proc Natl Acad Sci USA 102: 12956-12961.

Barrientos RM, Higgins EA, Sprunger DB, Watkins LR, Rudy JW, Maier SF (2002). Memory for context is impaired by a post context exposure injection of interleukin-1 beta into dorsal hippocampus. Behav Brain Res 134: 291-298. 
Barrientos RM, Sprunger DB, Campeau S, Higgins EA, Watkins LR, Rudy JW et al (2003). Brain-derived neurotrophic factor mRNA downregulation produced by social isolation is blocked by intrahippocampal interleukin-1 receptor antagonist. Neuroscience 121: 847-853.

Barrientos RM, Sprunger DB, Campeau S, Watkins LR, Rudy JW, Maier SF (2004). BDNF mRNA expression in rat hippocampus following contextual learning is blocked by intrahippocampal IL-1beta administration. J Neuroimmunol 155: 119-126.

Bartolomucci A, Palanza P, Parmigiani S, Pederzani T, Merlot E, Neveu PJ (2003). Chronic psychosocial stress down-regulates central cytokines mRNA. Brain Res Bull 62: 173-178.

Ben Hur T, Ben Menachem O, Furer V, Einstein O, Mizrachi-Kol R, Grigoriadis N (2003). Effects of proinflammatory cytokines on the growth, fate, and motility of multipotential neural precursor cells. Mol Cell Neurosci 24: 623-631.

Ben Hur T, Rogister B, Murray K, Rougon G, Dubois-Dalcq M (1998). Growth and fate of PSA-NCAM+ precursors of the postnatal brain. J Neurosci 18: 5777-5788.

Besedovsky HO, del-Rey A (1996). Immune-neuro-endocrine interactions: facts and hypotheses. Endocr Rev 17: 64-102.

Bianchi M, Fone KF, Azmi N, Heidbreder CA, Hagan JJ, Marsden CA (2006). Isolation rearing induces recognition memory deficits accompanied by cytoskeletal alterations in rat hippocampus. Eur J Neurosci 24: 2894-2902.

Bradbury EJ, Kershaw TR, Marchbanks RM, Sinden JD (1995). Astrocyte transplants alleviate lesion induced memory deficits independently of cholinergic recovery. Neuroscience 65: 955-972.

Brown RF, Tennant CC, Sharrock M, Hodgkinson S, Dunn SM, Pollard JD (2006). Relationship between stress and relapse in multiple sclerosis: part I. Important features. Mult Scler 12: 453-464.

Cao QL, Zhang YP, Howard RM, Walters WM, Tsoulfas P (2001). Pluripotent stem cells engrafted into the normal or lesioned adult rat spinal cord are restricted to a glial lineage. Exp Neurol 16: $48-58$

Davidson TL, Kanoski SE, Walls EK, Jarrard LE 2005. Memory inhibition and energy regulation. Physiol Behav 15: 731-746.

Depino AM, Alonso M, Ferrari C, del Ray A, Anthony D, Besedoysky $\mathrm{H}$ et al (2004). Learning modulation by endogenous hippocampal IL-1: blockade of endogenous IL-1 facilitates memory formation. Hippocampus 14: 526-535.

Dinarello CA (1996). Biologic basis for interleukin-1 in disease. Blood 87: 2095-2147.

Dinkel K, MacPherson A, Sapolsky RM (2003). Novel glucocorticoid effects on acute inflammation in the CNS. J Neurochem 84: 705-716.

Dong H, Goico B, Martin M, Csernansky CA, Bertchume A, Csernansky JG (2004). Modulation of hippocampal cell proliferation, memory, and amyloid plaque deposition in APPsw (Tg2576) mutant mice by isolation stress. Neuroscience 127: 601-609.

Ekdahl CT, Claasen JH, Bonde S, Kokaia Z, Lindvall O (2003). Inflammation is detrimental for neurogenesis in adult brain. Proc Natl Acad Sci USA 100: 13632-13637.

Emsley HC, Smith CJ, Georgiou RF, Vail A, Hopkins SJ, Rothwell NJ et al (2005). Acute stroke investigators. a randomised phase II study of interleukin-1 receptor antagonist in acute stroke patients. J Neurol Neurosurg Psychiatry 76: 1366-1372.

Fischer K, Mackensen A (2003). The flow cytometric PKH-26 assay for the determination of T-cell mediated cytotoxic activity. Methods 31: 135-142.

Gayle D, Ilyin SE, Plata-Salamán CR (1997). Central nervous system IL-1 beta system and neuropeptide Y mRNAs during IL-1 beta-induced anorexia in rats. Brain Res Bull 44: 311-317.

Goshen I, Kreisel T, Ben-Menachem-Zidon O, Licht T, Weidenfeld $\mathrm{J}$, Ben-Hur T et al (2007). Brain interleukin-1 mediates chronic stress-induced depression in mice via adrenocortical activation and hippocampal neurogenesis suppression. Mol Psychiatry [E-pub ahead of print].

Goshen I, Kreisel T, Ounallah-Saad H, Zalzstein Y, Ben-Hur T, Levy-Lahad $\mathrm{E}$ et al A dual role for interleukin-1 in hippocampal-dependent memory processes. Psychoneuroendocrinology (in press).

Goshen I, Yirmiya R (2007). The role of pro-inflammatory cytokines in memory processes and neural plasticity.In: Ader $\mathrm{R}$ (ed). Psychoneuroimmunology, 4th ed. Elsevier AP: USA, pp 337-377.

Griffin WS, Mrak RE (2002). Interleukin-1 in the genesis and progression of and risk for development of neuronal degeneration in Alzheimer's disease. J Leukoc Biol 72: 233-238.

Guo M, Wu CF, Liu W, Yang JY, Chen D (2004). Sex difference in psychological behavior changes induced by long-term social isolation in mice. Prog Neuropsychopharmacol Biol Psychiatry 28: $115-121$.

Hagan P, Barks JD, Yabut M, Davidson BL, Roessler B, Silverstein FS (1996). Adenovirus-mediated over-expression of interleukin1 receptor antagonist reduces susceptibility to excitotoxic brain injury in perinatal rats. Neuroscience 75: 1033-1045.

Harvey J (2003). Novel actions of leptin in the hippocampus. Ann Med 35: 197-206.

Heldt SA, Stanek L, Chhatwal JP, Ressler KJ (2007). Hippocampusspecific deletion of BDNF in adult mice impairs spatial memory and extinction of aversive memories. Mol Psychiatry 12: 656-670.

Holguin A, Frank MG, Biedenkapp JC, Nelson K, Lippert D, Watkins LR et al (2007). Characterization of the temporo-spatial effects of chronic bilateral intrahippocampal cannulae on interleukin-1beta. J Neurosci Methods 161: 265-272.

Hosoi T, Okuma Y, Nomura Y (2002). Leptin regulates interleukin1beta expression in the brain via the STAT3-independent mechanisms. Brain Res 949: 139-146.

Hovakimyan M, Haas SJ, Schmitt O, Gerber B, Wree A, Andressen C (2006). Mesencephalic human neural progenitor cells transplanted into the neonatal hemiparkinsonian rat striatum differentiate into neurons and improve motor behaviour. J Anat 209: 721-732.

Iguchi F, Nakagawa T, Tateya I, Kim TS, Endo T, Taniguchi Z et al (2003). Trophic support of mouse inner ear by neural stem cell transplantation. Neuroreport 14: 77-80.

Jacobsen JP, Mork A (2006). Chronic corticosterone decreases brain-derived neurotrophic factor (BDNF) mRNA and protein in the hippocampus, but not in the frontal cortex, of the rat. Brain Res 1110: 221-225.

Jeong YH, Park CH, Yoo J, Shin KY, Ahn SM, Kim HS et al (2006). Chronic stress accelerates learning and memory impairments and increases amyloid deposition in APPV717I-CT100 transgenic mice, an Alzheimer's disease model. FASEB J 20: 729-731.

Kempermann G, Kuhn H, Gage H (1997). Genetic influence on neurogenesis in the dentate gyrus of adult mice. Proc Natl Acad Sci USA 94: 10409-10414.

Kogan JH, Frankland PW, Silva AJ (2000). Long-term memory underlying hippocampus-dependent social recognition in mice. Hippocampus 10: 47-56.

Lee J, Duan W, Mattson MP (2002). Evidence that brain-derived neurotrophic factor is required for basal neurogenesis and mediates, in part, the enhancement of neurogenesis by dietary restriction in the hippocampus of adult mice. J Neurochem 82: 1367-1375.

Lee KJ, Kim SJ, Kim SW, Choi SH, Shin YC, Park SH et al (2006). Chronic mild stress decreases survival, but not proliferation, of new-born cells in adult rat hippocampus. Exp Mol Med 38: 44-54.

Loddick SA, Liu C, Takao T, Hashimoto K, De Souza EB (1998). Interleukin-1 receptors: cloning studies and role in central nervous system disorders. Brain Res Rev 26: 306-319. 
Lundkvist J, Sundgren-Andersson AK, Tingsborg S, Ostlund P, Engfors C, Alheim K et al (1999). Acute-phase responses in transgenic mice with CNS overexpression of IL-1 receptor antagonist. Am J Physiol 276: 644-651.

Lynch MA (2002). Interleukin-1 beta exerts a myriad of effects in the brain and in particular in the hippocampus: analysis of some of these actions. Vitam Horm 64: 185-219.

Monje ML, Toda H, Palmer TD (2003). Inflammatory blockade restores adult hippocampal neurogenesis. Science 302: 1760-1765.

Moynagh PN (2005). The interleukin-1 signalling pathway in astrocytes: a key contributor to inflammation in the brain. J Anat 207: 265-269.

O'Connor KA, Johnson JD, Hansen MK, Wieseler Frank JL, Maksimova E, Watkins LR et al (2003). Peripheral and central proinflammatory cytokine response to a severe acute stressor. Brain Res 991: 123-132.

Plata-Salaman CR, Ilyin SE, Turrin NP, Gayle D, Flynn MC, Bedard $\mathrm{T}$ (2000). Neither acute nor chronic exposure to a naturalistic (predator) stressor influences the interleukin-1beta system, tumor necrosis factor-alpha, transforming growth factor-betal, and neuropeptide mRNAs in specific brain regions. Brain Res Bull 51: 187-193.

Pluchino S, Zanotti L, Martino G (2007). Rationale for the use of neural stem/precursor cells in immunemediated demyelinating disorders. J Neurol 254: I23-I28.

Pollak Y, Ovadia H, Orion E, Weidenfeld J, Yirmiya R (2003a). The EAE-associated behavioral syndrome: I. Temporal correlation with inflammatory mediators. J Neuroimmunol 137: 94-99.

Pollak Y, Ovadia H, Orion E, Yirmiya R (2003b). The EAEassociated behavioral syndrome: II. Modulation by anti-inflammatory treatments. J Neuroimmunol 137: 100-108.

Pugh CR, Fleshner M, Watkins LR, Maier SF, Rudy JW (2001). The immune system and memory consolidation: a role for the cytokine IL-1beta. Neurosci Biobehav Rev 25: 29-41.

Pugh CR, Johnson JD, Martin D, Rudy JW, Maier SF, Watkins LR (2000). Human immunodeficiency virus-1 coat protein gp120 impairs contextual fear conditioning: a potential role in AIDS related learning and memory impairments. Brain Res 861: 8-15.

Pugh CR, Kumagawa K, Fleshner M, Watkins LR, Maier SF, Rudy JW (1998). Selective effects of peripheral lipopolysaccharide administration on contextual and auditory-cue fear conditioning. Brain Behav Immun 12: 212-229.

Pugh CR, Nguyen KT, Gonyea JL, Fleshner M, Wakins LR, Mair SF et al (1999). Role of interleukin-1 beta in impairment of contextual fear conditioning caused by social isolation. Behav Brain Res 106: 109-118.

Quan N, He L, Lai W (2003). Intraventricular infusion of antagonists of IL-1 and TNF alpha attenuates neurodegeneration induced by the infection of Trypanosoma brucei. J Neuroimmunol 138: 92-98.

Rothwell NJ, Luheshi GN (2000). Interleukin 1 in the brain: biology, pathology and therapeutic target. Trends Neurosci 23: 618-625.

Saxe MD, Battaglia F, Wang JW, Malleret G, David DJ, Monckton JE et al (2006). Ablation of hippocampal neurogenesis impairs contextual fear conditioning and synaptic plasticity in the dentate gyrus. Proc Natl Acad Sci USA 103: 17501-17506.
Scaccianoce S, Del Bianco P, Paolone G, Caprioli D, Modafferi AM, Nencini $\mathrm{P}$ et al (2006). Social isolation selectively reduces hippocampal brain-derived neurotrophic factor without altering plasma corticosterone. Behav Brain Res 168: 323-325.

Schneider H, Pitossi F, Balschum D, Wagner A, Del Rey A, Beseddovsky H (1998). A neuromodulatory role of interleukin-1beta in the hippocampus. Proc Natl Acad Sci USA 95: 7778-7783.

Shors TJ (2004). Memory traces of trace memories: neurogenesis, synaptogenesis and awareness. Trends Neurosci 27: 250-256.

Shors TJ, Miesegaes G, Beylin A, Zhao M, Rydel T, Gould E (2001). Neurogenesis in the adult is involved in the formation of trace memories. Nature 410: 372-376.

Shors TJ, Townsend DA, Zhao M, Kozorovitskiy Y, Gould E (2002). Neurogenesis may relate to some but not all types of hippocampal-dependent learning. Hippocampus 12: 578-584.

Simon M, Czeh B, Fuchs E (2005). Age-dependent susceptibility of adult hippocampal cell proliferation to chronic psychosocial stress. Brain Res 1049: 244-248.

Srinivasan D, Yen JH, Joseph DJ, Friedman W (2004). Cell typespecific interleukin-1beta signaling in the CNS. J Neurosci 24 6482-6488.

Stranahan AM, Khalil D, Gould E (2006). Social isolation delays the positive effects of running on adult neurogenesis. Nat Neurosci 9: 526-533.

Svendsen CN, Borg MG, Armstrong RJ, Rosser AE, Chandran S, Ostenfeld $\mathrm{T}$ et al (1998). A new method for the rapid and long term growth of human neural precursor cells. J Neurosci Methods 85: 141-152.

Wada A, Yokoo H, Yanagita T, Kobayashi H (2005). New twist on neuronal insulin receptor signaling in health, disease, and therapeutics. J Pharmacol Sci 99: 128-143.

Westenbroek C, Den Boer JA, Veenhuis M, Ter Horst GJ (2004). Chronic stress and social housing differentially affect neurogenesis in male and female rats. Brain Res Bull 64: 303-308.

Wilson RS, Evans DA, Bienias JL, Mendes de Leon CF, Schneider JA, Bennett DA (2003). Proneness to psychological distress is associated with risk of Alzheimer's disease. Neurology 61: $1479-1485$

Winocur G, Wojtowicz JM, Sekeres M, Snyder JS, Wang S (2006). Inhibition of neurogenesis interferes with hippocampus-dependent memory function. Hippocampus 16: 296-304.

Wolf G, Yirmiya R, Goshen I, Iverfeldt K, Holmlund L, Takeda K et al (2003). Impairment of interleukin-1 (IL-1) signaling reduces basal pain sensitivity in mice: genetic, pharmacological and developmental aspects. Pain 104: 471-480.

Yirmiya R, Pollak Y, Morag M, Reichenberg A, Barak O, Avitsur R et al (2000). Illness, cytokines and depression. Ann NY Acad Sci 917: 478-487.

Yirmiya R, Weidenfeld J, Barak O, Avitsur R, Pollak Y, Gallily R et al (1999). The role of brain cytokines in mediating the behavioral and neuroendocrine effects of intracerebral mycoplasma fermentans. Brain Res 829: 28-38.

Yirmiya R, Winocur G, Goshen I (2002). Brain interleukin-1 is involved in spatial memory and passive avoidance conditioning. Neurobiol Learn Mem 78: 379-389. 